\title{
On Open Membranes, Cosmic Strings and Moduli Stabilization
}

\author{
Evgeny I. Buchbinder \\ School of Natural Sciences, Institute for Advanced Study \\ Einstein Drive, Princeton, NJ 08540
}

\begin{abstract}
We discuss how cosmic strings can be created in heterotic M-theory compactifications with stable moduli. We conclude that the only appropriate candidates seem to be fundamental open membranes with a small length. In four dimensions they will appear as strings with a small tension. We make an observation that, in the presence of the vector bundle moduli, it might be possible to stabilize a five-brane very close to the visible sector so that a macroscopic open membrane connecting this five-brane and the visible brane will have a sufficiently small length. We also discuss how to embed such cosmic strings in heterotic models with stable moduli and whether they can be created after inflation.
\end{abstract}




\section{Introduction}

String theory seems to have appropriate candidates for cosmic strings. The possibility of viewing superstrings as cosmic strings was first discussed by Witten in [1]. He pointed out some serious problems in this direction. For example, one of the common problems is that the tension of fundamental superstrings is much bigger than the observational bound on the cosmic string tension. Another very common problem is that cosmic superstrings appear as boundaries of axion domain walls. This will force the strings to collapse after the axion gets a mass [2].

Cosmic strings in the context of string theory were recently reconsidered in [3, 4, 5, 6]. It was argued that the previous problems might be avoided thanks to recent developments in string theory, including D-, NS- and M-branes, compactifications with large warp factors and models with large extra dimensions. In particular, small tension of cosmic superstrings can be explained by a large warp factor. A systematic analysis of fundamental and Dirichlet cosmic strings was performed by Copeland, Myers and Polchinski in [6]. Results obtained in [6] suggest that in type IIB compactifications (at least in models with many Klebanov-Strassler throats [7]) it is possible to have brane/anti-brane inflation [8 followed by production of metastable F- and D-strings and de Sitter (dS) vacua 9]. Furthermore, it was proposed in 10, 11, 12 that cosmic D-strings can be identified with solitons in supergravity with Fayet-Iliopoulos (FI) terms. A holographic dual of D-strings in the throat was studied in $[13$.

The most phenomenologically attractive four-dimensional vacua arise in string/M-theory from compactifications of strongly coupled heterotic string [14, 15, 16]. Recently, it was demonstrated [17, 18] that heterotic string theory on smooth non-simply connected CalabiYau manifold with $Z_{3} \times Z_{3}$ homotopy group can give rise to a four-dimensional theory whose spectrum differs form the spectrum of the Standard Model only by one extra Higgs multiplet. A progress has also been made in heterotic models towards moduli stabilization [19, 20, 21, 22, 23] and inflation [24, 25]. In this paper, we ask a question whether it is possible to find cosmic strings in heterotic compactifications with stable moduli. In section 2, we discuss several ways how cosmic strings could be created and come to conclusion that the only candidates seem to be fundamental open membranes with a small length. In four dimensions,

an open membrane appears as a string. Such membranes could arise if one of five-branes is stabilized close to one of the orbifold fixed planes. In section 3, we show that it might be possible to stabilize a five-brane close to the visible brane due to its non-perturbative 
interaction with the vector bundle moduli. In section 4, we discuss stabilization of the remaining moduli. We slightly generalize the earlier results [20, 23] and point out that it should be possible to stabilize the interval in the phenomenological range in the presence of many five-branes in the bulk without any extra assumptions. Cosmic strings are unstable in the presence of axion domain walls. In [6], it was shown that axion domain walls will not be formed if the axion is charged under a $U(1)$ gauge group. In heterotic string theory this happens if in four dimensions there is an anomalous $U(1)$ whose anomaly is canceled by the Green-Schwarz mechanism [26]. However, to keep this argument valid, it is important that the axion does not receive any potential. Since in heterotic M-theory the axion is the imaginary part of the volume multiplet [27, 28], no superpotential for the volume should be present. This makes it difficult to stabilize the volume. Fortunately, the presence of the anomalous $U(1)$ implies the presence of volume-dependent FI-terms. We show that together with the F-terms, the FI-terms naturally provide stabilization of all moduli in a non-supersymmetric Anti de Sitter (AdS) vacuum. In subsection 4.2, we speculate how cosmic strings can be embedded into compactifications with dS vacua. We show that it might be possible in non-supersymmetric compactifications. In section 5 , we discuss how the cosmic strings, studied in this paper, can be created after inflation.

\section{Cosmic Strings in Heterotic M-theory}

In this section, we will point out that it is harder to find string-like objects in Calabi-Yau compactifications of heterotic M-theory comparing to type II compactifications. Let us start with discussing whether cosmic strings can arise after inflation considered in [24]. The inflationary phase in [24] is represented by a five-brane, wrapped on an non-isolated genus zero or a higher genus holomorphic curve in a Calabi-Yau threefold, approaching the visible brane ${ }^{1}$. It was argued in 24] that as the five-brane comes too close the transition vector bundle moduli [29, 30] associated with this holomorphic curve become tachyonic terminating inflation. In the post-inflationary phase, the five-brane disappears (becomes massive) through the small instanton transition [31, 32, 33, whereas the transition vector bundle moduli can be stabilized by non-perturbative effects. Thus, the post-inflationary physics does not contain any rolling moduli. All the remaining moduli are stabilized both during and after inflation. The cosmological constant, generically, changes after the small instanton transition. This

\footnotetext{
${ }^{1}$ Throughout the paper, we will refer to one of the orbifold fixed planes as to the visible brane, or the visible sector, and to the other one as to the hidden brane, or the hidden sector.
} 
leads to the possibility of obtaining dS vacua with a small cosmological constant in the post-inflationary phase. A five-brane wrapped on a Riemann surface of genus one or higher carries one or more $U(1)$ gauge fields on its world-volume 34]. For simplicity, consider a five-brane wrapping a torus so that there is only one $U(1)$ factor. During the small instanton transition, this $U(1)$ gets broken. Therefore, it seems conceivable that cosmic strings can be created by the Kibble mechanism [35. However, it is not the case. First problem is that the small instanton transition may not be describable by field theory, whereas the Kibble argument is based on the Lagrangian description. Second, even if there is a field theory description of the small instanton transition (one might expect it if a Calabi-Yau threefold is not simply connected), one should recall that the theory we deal with is the $S^{1} / Z_{2}$ orbifold. Therefore, when a five-brane coincides with one of the orbifold fixed plains, it also coincides with its image. In this case, one should expect that the gauge group which gets restored is $S U(2)$. As a result, after the small instanton transition the group which is broken is $S U(2)$ and no strings are formed. This set-up has one more candidate for cosmic string in the vector-bundle branch. When a five-brane coincides with an orbifold fixed plane, a tensionless string arises [36]. Can it get a tension in the vector bundle branch, with its tension being set by the vector-bundle moduli? The answer is again negative. This tensionless string should be understood as the analog of a monopole becoming light. This analogy suggests that this string will get a tension in the five-brane branch. In the vector bundle branch, it will not exist for the same reason why monopoles do not exist in the Higgs branch. This discussion demonstrates that no cosmic strings are formed after inflation presented in [24]. Below, in the paper, we will modify the set-up of [24] to allow cosmic strings to be formed after inflation.

Let us now discuss whether it is possible to create cosmic strings after a hypothetical (not yet constructed) five-brane/anti-five-brane inflation. After five-brane/anti-five-brane annihilation, a membrane bound state can be formed [37. This membrane will be parallel to the orbifold fixed planes. If our Calabi-Yau threefold is non-simply connected, one can wrap such a membrane on a one-cycle with non-trivial $\pi_{1}$ and form a string. However, this string will be stable in M-theory but not in heterotic M-theory. A membrane parallel to the orbifold fixed planes is not supersymmetric [38, 39]. Therefore, one should expect that it will quickly annihilate with its image.

Our discussion shows that it is hard to find cosmic strings in heterotic M-theory. However, we have not yet considered the most natural candidate, a fundamental open membrane. From the four-dimensional viewpoint such a membrane looks like a string. The tension of such a 
string behaves as

$$
\mu \sim M_{11}^{3} y
$$

where $M_{11}$ is the eleven-dimensional Planck scale and $y$ is the length of the membrane in the eleventh dimension. It is easy to estimate that in order to fulfill the observational bound $G \mu<10^{-7}-10^{-6}$ (see, for example, 40] and references therein), the ratio of $y$ to the size of the interval $\pi \rho$ should be of order

$$
\frac{y}{\pi \rho} \sim 10^{-4}-10^{-3}
$$

To obtain (2.2), we set the volume scale $v_{C Y}$ and the interval scale $\pi \rho$ to be in the phenomenological range, $v_{C Y}^{1 / 6} \sim\left(10^{16} \mathrm{GeV}\right)^{-1}$ and $\pi \rho \sim\left(10^{15} \mathrm{GeV}\right)^{-1}$ and used the fact that the eleven-dimensional and four-dimensional Planck scales, $M_{11}$ and $M_{P l}$, are related as follows

$$
M_{P l}^{2}=M_{11}^{9} v_{C Y} \pi \rho
$$

Production of strings with such a tension implies some constraints on the five-brane stabilization. One of the five-branes in the bulk has to be stabilized very close to either one of the orbifold fixed planes or another five-brane. In the next section, we will argue that it is possible to stabilize a five-brane close to the visible brane. The key role in this process is played by the vector bundle moduli and their interaction. In later sections, we will extend results of section 3 to include the remaining moduli and the inflaton.

\section{Five-brane Stabilization and Open Membranes with a Small Length}

We consider a Calabi-Yau compactification of heterotic M-theory with the following complex moduli

$$
S, \quad T^{I}, \quad Y, \quad Z_{\alpha} ; \quad Y_{0}, \phi_{i} .
$$

Here $S=V+i \sigma$, where $V$ is the volume modulus and $a$ is the axion, $T^{I}$ 's are the Kahler moduli. They are constructed as follows [27, 28]

$$
T^{I}=R b^{I}+i p^{I}, \quad I=1, \ldots, h^{1,1}
$$

where $R$ is the interval modulus, $b^{I}$ are the $(1,1)$ moduli of the Calabi-Yau threefold and $p^{I}$ 's come from the components of the M-theory three-form $C$ along the interval and the CalabiYau manifold. The moduli $Y$ and $Y_{0}$ correspond to five-branes wrapped on an isolated genus 
zero holomorphic curve in the Calabi-Yau threefold. The real part of each of the two fivebrane multiplets is the position of the corresponding five-brane in the interval. We denote them by $y$ and $y_{0}$ respectively. The imaginary part is related to the axions on the five-brane worldvolume. See 41 for details. One five-brane is needed to stabilize the interval in a phenomenological range as in 20, [23. This five-brane has to be located relatively close to the hidden brane. The other five-brane will be the one that we will attempt to stabilize in this section close to visible brane so that eq. (2.2) is satisfied. $Z_{\alpha}$ are the complex structure moduli, whose actual number is irrelevant. We will assume that the $(3,0,1)$ flux $G$ is turned on. Such a flux produces the superpotential for $Z_{\alpha}$ of the form [42, 43]

$$
W_{f}=\frac{M_{P l}^{2}}{v_{C Y} \pi \rho} \int d x^{11} \int_{C Y} G \wedge \Omega .
$$

This superpotential is expected, generically, to stabilize all the complex structure moduli. We will assume that it is the case. We will also assume that the complex structure moduli are stabilized at slightly higher scale that all the other moduli so that $W_{f}$ can be considered as constant in the low-energy field theory. At last, $\phi_{i}$ are the moduli of the vector bundle on the visible brane. For simplicity, we will assume that the bundle on the hidden brane either is trivial or does not have any moduli. The moduli $V$ and $R$ are assumed to be dimensionless and normalized with respect to the reference scales $v_{C Y} \sim\left(10^{16} \mathrm{GeV}\right)^{-1}$ and $R \sim\left(10^{15} \mathrm{GeV}\right)^{-1}$. The moduli $y$ and $y_{0}$ are also dimensionless and less that $R$. To obtain the four-dimensional coupling constants in a phenomenological range, $V$ and $R$ have to be stabilized at (or be slowly rolling near) a value of order one.

In this section, we will concentrate on the moduli $Y_{0}$ and $\phi$ assuming that all the other moduli are stable. They will be considered in the next section. The Kahler potential of the system (in the four-dimensional Planck units) is given by

$$
K\left(Y_{0}, \phi\right)=K_{0}+K_{1}\left(Y_{0}+\bar{Y}_{\mathbf{0}}\right)^{2}+k K(\phi)
$$

Here $K_{0}$ and $K_{1}$ are functions of the remaining moduli independent of $Y_{0}$ whose precise form is irrelevant for us. $K(\phi)$ is the Kahler potential for the vector bundle moduli $\phi_{i}$ and $k$ is a dimensionless parameter of order $10^{-5}$ [20]. It is much less than one because the gauge sector in Horava-Witten theory [14, 15] arises at the sub-leading order in the gravitational coupling constant comparing to the gravity sector. The superpotential (in the four-dimensional Planck units) is given by

$$
W=W_{0}+\alpha \operatorname{Pfaff}\left(\mathcal{D}_{-}\right) e^{-\tau Y_{0}}
$$


Here the second term is the non-perturbative superpotential due to a membrane instanton [44, 45, 46, 38, 39, 47, 48, with $\alpha$ being a dimensionless parameter, and $W_{0}$ is the superpotential depending on the remaining moduli. For our purposes it is a constant. The parameter $\tau$ is given by

$$
\tau=\frac{1}{2}(\pi \rho) v\left(\frac{\pi}{2 \kappa_{11}}\right)^{1 / 3}
$$

where $v$ is the area of the holomorphic curve on which the membrane is wrapped. Generically, it is of order 100. $\operatorname{Pfaff}\left(\mathcal{D}_{-}\right)$is the Pfaffian of the Dirac operator depending on the vector bundle moduli. It is very difficult to compute it explicitly because no gauge connections on Calabi-Yau threefolds are known. However, in [47, 48, this factor was computed in a number of examples for the case the Calabi-Yau threefold elliptically fibered over the Hirzebruch surface using algebraic geometry techniques. It was found to be a high degree homogeneous polynomial in the vector bundle moduli. We will denote it as $\mathcal{P}(\phi)$. If the fivebrane is close to the visible sector, all other $\phi_{i}$ and $Y_{0}$ contributions to the non-perturbative superpotential are negligibly small. If we ignore all other moduli (they will be discussed later), the minimum of the potential energy for the $Y_{0}, \phi_{i}$ system is given by

$$
D_{\phi_{i}} W=0, \quad D_{Y_{0}} W=0,
$$

where $D W$ is the Kahler covariant derivative, $D W=\partial W+\frac{W}{M_{P l}^{2}} \partial K$. From eqs. (3.4) and (3.5) we have

$$
D_{\phi_{i}} W=\frac{\partial \mathcal{P}(\phi)}{\partial \phi_{i}} e^{-\tau Y_{0}}+k \frac{\partial K(\phi)}{\partial \phi_{i}} W=0
$$

and

$$
D_{Y_{0}} W=-\tau \mathcal{P}(\phi) e^{-\tau Y_{0}}+4 K_{1} y_{0} W=0 .
$$

It is easy to realize that since $\tau$ is much bigger than one and $k$ is much less than one, in the second terms of eqs. (3.8) and (3.9), we can replace $W$ with $W_{0}$. If the degree of the polynomial $\mathcal{P}(\phi)$ is sufficiently high, the first terms is eqs. (3.8) and (3.9) are practically the same. The second term in eq. (3.8) is proportional to $k$ whereas the second term in eq. (3.9) is proportional to $y_{0}$. These two equations are consistent only if $y_{0} \propto k$, that is $y_{0}$ is much less than one. Whether or not the solution for $y_{0}$ is consistent with (2.2) depends on details of the compactification, in particular, on the Kahler potential $K(\phi)$ which is unknown how to evaluate explicitly on a Calabi-Yau threefold. Nevertheless, the form of eqs. (3.8) and (3.9) suggests that in some models it might be possible to find a solution for $y_{0}$ consistent with (2.2). 
To get a better understanding how a solution for $y_{0}$ might look like, let us make a reasonable model for the Kahler potential $K(\phi)$. Let us choose, for simplicity, $K(\phi)$ to be flat

$$
K(\phi)=\sum_{i=1}\left|\phi_{i}\right|^{2}
$$

It corresponds to the Kahler potential for the size and orientation moduli of centered YangMills instantons on $\mathbb{R}^{4}$ if the number of moduli $\phi_{i}$ is even [49, 50, 51]. The Pfaffian will be assumed to be a generic homogeneous polynomial of degree $d$. It is obvious that for a generic Pfaffian it is possible to find a solution for the phases of $\phi$ 's and the imaginary part of $Y_{0}$. Let $r_{i}$ be the absolute value of $\phi_{i}$. Consider eqs. (3.8) for $r_{i}$. Since we are interested in solutions with $\tau y_{0}<<1$, the exponential factor $e^{-\tau y_{0}}$ in eqs. (3.8), (3.9) can be set to unity. Then using eq. (3.10), eqs. (3.8) for $r_{i}$ can be written as follows

$$
\mathcal{F}_{i}\left(r_{j}\right)=\frac{k\left|W_{0}\right|}{\alpha d}
$$

where each $\mathcal{F}_{i}$ is a homogeneous function of $r_{j}$ of degree $d-2$. The factor $d$ in the denominator in the left hand side comes form differentiating the Pfaffian ${ }^{2}$. From eqs (3.11) it follows that every $r_{i}$ is of the form

$$
r_{i} \propto\left(\frac{k\left|W_{0}\right|}{\alpha d}\right)^{1 /(d-2)},
$$

where the proportionality coefficient is a number depending on details of the Pfaffian. Then from eq. (3.9) it follows that

$$
y_{0} \sim \frac{\alpha \tau}{4 K_{1}\left|W_{0}\right|}\left(\frac{k\left|W_{0}\right|}{\alpha d}\right)^{d /(d-2)}
$$

If $d>>1$, we have

$$
y_{0} \sim \frac{\tau k}{4 K_{1} d} .
$$

Taking, for example, $k \sim 10^{-5}, \tau \sim 100, d \sim 10,4 K_{1} \sim 1$, we find that

$$
y_{0} \sim 10^{-4}
$$

which is consistent with condition (2.2). Note that $y_{0}$ is inverse proportional to $d$. A large value of $d$, which is consistent with results of [47, 48], might also be a reason for a small value of $y_{0}$.

In this section, we demonstrated that it is possible to stabilize a five-brane, wrapped on an isolated genus zero holomorphic curve, close to the visible brane. From the four-dimensional

\footnotetext{
${ }^{2}$ Without loss of generality, we can assume that the Pfaffian contains terms $r_{j}^{d}$.
} 
viewpoint, a macroscopic open membrane stretched between this five-brane and the visible brane will look like a cosmic string with a relatively small tension which might be consistent with the observational bound.

\section{Stabilization of the Remaining Moduli}

\subsection{AdS Vacua}

In this section, we will discuss how cosmic strings from section 3 can be embedded into compactifications with stable moduli. For this we need to stabilize the remaining moduli in (3.1), that is $S, T^{I}$ and $Y$. The moduli $T^{I}$ and $Y$ can be stabilized by the same methods as in [20, 23]. However, stabilization of $S$ represents a problem. The imaginary part of $S$ is the axion. It has been known that cosmic strings of the type constructed in the previous section are boundaries of axion domain walls [1]. If the axion gets a mass the strings become unstable 2]. This problem can be avoided if the axion is charged under some $U(1)$ [6]. In heterotic M-theory such a situation occurs if there is an anomalous $U(1)$ in four dimensions. The anomaly of such a $U(1)$ is canceled by the four-dimensional version of the Green-Schwarz mechanism. The axion, in this case, transforms under $U(1)$ as

$$
\delta \sigma=\lambda
$$

Now the axion becomes a pure gauge degree of freedom and can be gauged away. As a result, no axion domain walls are formed. The above arguments will become invalid if the axion receives a potential. This, in particular, indicates that stability of cosmic strings requires that no superpotentials for the $S$ modulus should be present. An anomalous $U(1)$ whose presence, as we just explained, is important for stability of cosmic strings, gives rise to the moduli dependent FI-terms [26]. In heterotic M-theory they are of the form [23]

$$
\frac{U_{F I}}{M_{P l}^{4}} \sim \frac{g^{2}}{V^{2}} .
$$

Here $g$ is the gauge coupling constant which is itself moduli dependent

$$
g^{2}=\frac{g_{0}^{2}}{R e(S+\ldots)}
$$

and the ellipsis stands for the $T^{I}$ - and $Y$-dependent corrections. The precise form of the corrections depends whether an anomalous $U(1)$ is in the visible or in the hidden sector. They 
can be found, for example, in [34]. To simplify equations below, we will assume that they are small and can be ignored. Their presence can be shown not to lead to any conceptually new results below. The order of magnitude of $U_{F I}$ was estimated in [23] and was found to be, generically of order $\frac{W_{f}^{2}}{M_{P l}^{2}}$.

As we said, the moduli $T^{I}$ and $Y$ can be stabilized by methods presented in [20, 23. Let us briefly discuss them with a slight generalization. For simplicity, we will consider the case $h^{1,1}=1$. Generalizations for $h^{1,1}>1$ is straightforward but technically more complicated. In this case, there is only one $T$-modulus whose real part is the length of the interval. If the five-brane whose modulus has been denoted by $Y$ is located close to the hidden sector, the leading contribution to the superpotential $W_{0}$ is of the form

$$
W_{0}=W_{f}+\beta e^{-\tau(T-Y)} .
$$

The scale of the coefficient $\beta$ will be assumed to be set by the eleven-dimensional Planck scale. The equation of motion for $R$, schematically, is [20, 23]

$$
e^{-\tau(R-y)} \sim \frac{\left|W_{f}\right|}{\beta \tau}
$$

In order this equation to have a solution, the right hand side has to be less than one. First, $W_{f}$ is quantized in units of $\left(\frac{\kappa_{11}}{4 \pi}\right)^{2 / 3}$. Therefore, in the limit of large volume and large interval, the ratio $\frac{\left|W_{f}\right|}{\beta}$ is less than one. Second, the order of magnitude of $W_{f}$ might be reduced by Chern-Simons invariants [21]. Third, $\tau$ is much greater than one. This guarantees that, generically, the right hand side in eq. (4.5) is less than one. However, to stabilize $R$ in the phenomenological regime, $R \sim 1$, the five-brane $Y$ has to be stabilized close to the hidden sector so that $R-y$ is sufficiently small. Whether or not $Y$ can be stabilized close enough depends on details of the compactification. However, there is one obvious possibility of making $R-y$ small, which has not been considered before. Let us assume that in the bulk there are $N$ five-branes wrapped on the same holomorphic curve. Open membrane instantions will generate contributions to the superpotential inverse proportional to the exponent of the length between the five-branes. Then the equation of motion for the $i$-th five-brane will, schematically, be as follows

$$
-\beta_{i} e^{-\tau\left(y_{i}-y_{i-1}\right)}+\beta_{i+1} e^{-\tau\left(y_{i+1}-y_{i}\right)} \sim \frac{\left|W_{f}\right|}{\tau}
$$

Writing similar equations for all $N$ five-branes and the interval, it is possible to show that the five-branes and the interval will be stabilized in such a way that the distance between 
any two adjacent objects will approximately be the same and, therefore, of order $\frac{R}{N}$. Taking $N$ big enough, it is always possible to find a solution

$$
R \sim 1
$$

from eq. (4.5).

Let us point out that our analysis in section 3 and in the present section shows that a fivebrain in the bulk might have several different minima. In particular, there is a minimum close to the visible sector whose existence is due to the vector bundle moduli. On the other hand, there is a minimum at a generic point in the interval due to the non-perturbative exponential interaction with the adjacent branes (five-branes or the end-of -the-world branes) on the left and on the right. Putting $N$ five-branes will make the distance between any two adjacent branes of order $\frac{R}{N}$.

However, stabilization of the volume becomes a problem. In 20, 21, 23, the volume was stabilized by balancing the gaugino condensation superpotential against the fluxes. As we pointed out, any volume dependent superpotential is also a potential for the axion which will destabilize cosmic strings. Thus, in this paper, we will assume that no superpotential for the $S$-modulus is generated. In this subsection, we will consider the simplest potential for the volume generated by the Kahler potential and by the FI-terms. Note that existence of the FIterms is not an extra assumption. It already follows from existence of anomalous $U(1)$ which is necessary to gauge away the axion. The Kahler potential for $S$ is as follows [27, 28, 41]

$$
K(S)=-\ln (S+\bar{S})+\frac{c}{S+\bar{S}}
$$

where $c$ depends on $T^{I}$ - and $Y$-moduli which are assumed to be stable in this paper. To keep the metric in the $S, T^{I}, Y$ moduli space positive definite, at least in the large volume and the large interval limit, $c$ has to be sufficiently small so that the second term is sub-leading. Then by studying equations of motion for $V$, it is possible to realize that the second term in (4.8) does not play an important role in existence of extrema in the $V$-direction. Thus, for simplicity, we can assume that $c$ is sufficiently small and can be ignored to the leading order. It is also possible to show that the Kahler covariant derivatives for Kahler and five-brane moduli will be shifted from zero by terms proportional to $\frac{1}{\tau}$ which is much less than one. Thus, approximately, dynamics of $V$ is governed by the potential ${ }^{3}$

$$
U(V)=e^{K(S)}\left[G_{S \bar{S}}^{-1} D_{S} W D_{\bar{S}} \bar{W}-3 W \bar{W}\right]+U_{F I} \approx-\frac{A}{V}+\frac{B}{V^{3}} .
$$

\footnotetext{
${ }^{3}$ The FI terms will modify equations of motion for the interval and the five-branes. However, since the order of magnitude of FI terms is the same as that of the fluxes, a solution for these moduli will still exist.
} 


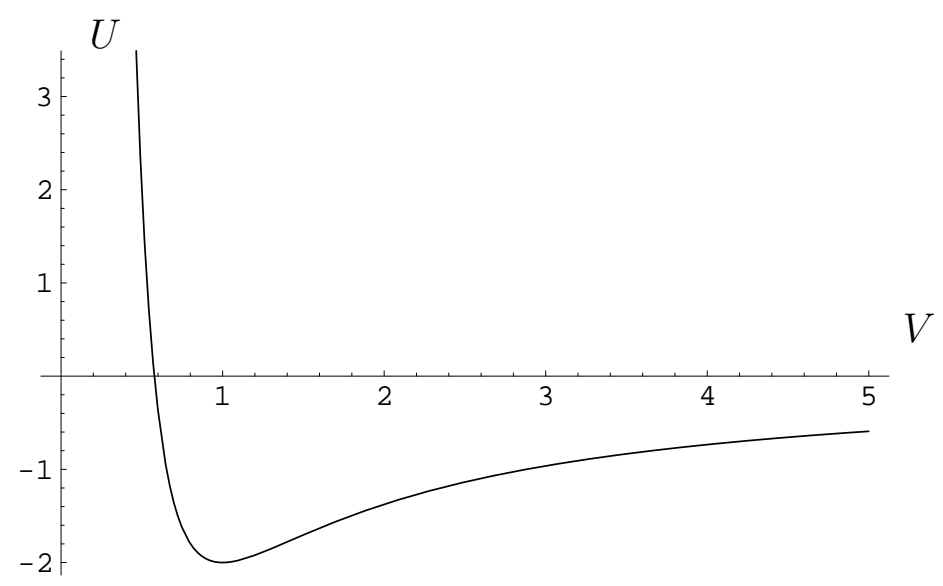

Figure 1: Potential $U(V)$ (appropriately normalized) for $A=3, B=1$. There is an AdS minimum.

Here $A$ and $B$ are constants (depending on vevs. of other moduli). The scale of $A$ is set by the fluxes and the scale of $B$ is set by the FI-terms. Generically, both $A$ and $B$ are of the same order of magnitude 23]. This potential has a non-supersymmetric AdS minimum. See fig. 1. Our discussion in this section shows that it is conceivable to find stable cosmic strings in heterotic compactifications with a stable non-supersymmetric AdS vacuum.

To conclude this subsection, let us make sure that addition of new moduli does not destabilize the moduli $\phi$ and $y_{0}$ from section 3 . It is easy to show that up to terms linear in $y_{0}$ the equation of motion for $\phi_{i}$ is still

$$
D_{\phi_{i}} W=0 .
$$

Similarly, taking into account the precise dependence of the FI-terms on $y_{0}$ [34, 23], one can show that eq. $D_{Y_{0}} W=0$ is modified by terms linear in $y_{0}$. This means that the form of eqs. (3.8) and (3.9) will not change and a solution $y_{0}<<1$ will still exist.

\section{2 dS Vacua?}

Let us now discuss how these results can be extended to compactifications with dS vacua. The most common strategy to obtain dS vacua [9, 52, 23] is to use the F-terms to obtain a supersymmetric AdS vacuum and then raise with extra terms (breaking supersymmetry) in the potential energy. However, as we discussed in the previous subsection, in the presence of cosmic strings it is problematic to obtain a supersymmetric AdS vacuum because we cannot use a superpotential for the volume multiplet. As a result, we had to use FI-terms to stabilize the volume rather than to raise an AdS vacuum. One way to create dS vacua 


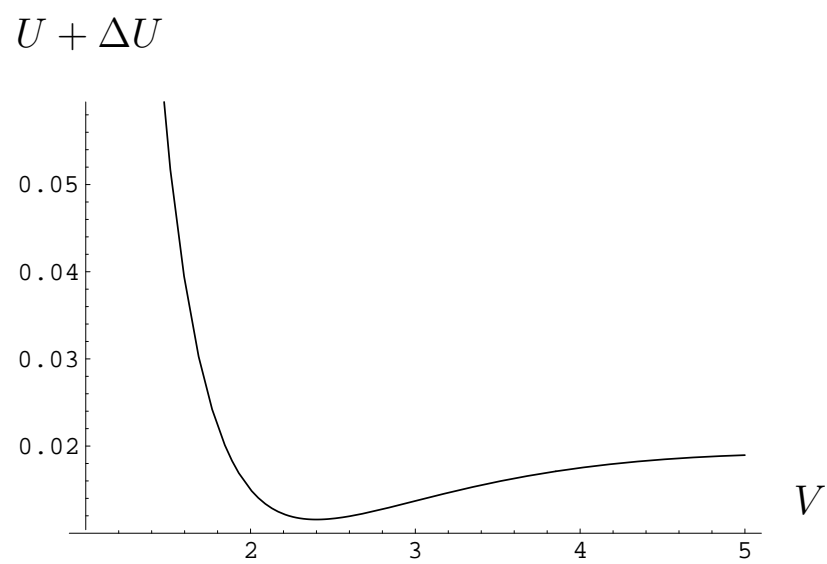

Figure 2: Potential $U(V)+\Delta U(V)$ (appropriately normalized) for $A=0.25, B=1.2, C=1$. There is a dS minimum.

can be to stabilize the volume by higher order corrections to the Kahler potential. This was studied recently in type IIB compactifications in [53, 54, 55]. However, it is not known how to extend these results to M-theory. One can also worry that, generically, this approach will stabilize the volume away from a phenomenologically interesting range. Another approach could be to search for extra corrections to the potential, in addition to the F-terms and the FI-terms. Such corrections arise, for example, in non-supersymmetric compactifications, which, in general, represent an approach for obtaining dS vacua [23, 56].

Below, we will show that under some conditions a dS vacuum can be found in nonsupersymmetric $E_{8} \times \bar{E}_{8}$ compactifications. In such compactifications, there is an extra volume-dependent contribution to the potential of the form 23 ]

$$
\Delta U(V)=\frac{a}{V}
$$

The coefficient $a$ depends on the $h^{1,1}$ moduli and on the interval. Generically, its order of magnitude is bigger than that of the fluxes and the FI-terms. However, it depends on the $h^{1,1}$ moduli. Here, we will assume that the $h^{1,1}$ moduli are stabilized in such a way that $\Delta U(V)$ is comparable with $U(V)$. It would be interesting to understand under what circumstances it is possible to stabilize a two-cycle at a very small size. We will not discuss it in this paper. Let us consider the potential $U(V)+\Delta U(V)$ including linear terms in $\frac{c}{V}$. It looks as follows

$$
U(V)+\Delta U(V)=\frac{A^{\prime}}{V}-\frac{C}{V^{2}}+\frac{B}{V^{3}} .
$$

Here $A^{\prime}$ is $a-A$. We will assume that it is positive. $C$ is linear in $c$. It is always positive and comes from the factor $e^{K(S)}$ in eq. (4.9). It is obvious that under some conditions on the parameters $A^{\prime}, B$ and $C$, this potential can have a metastable dS vacuum. See fig. 2. 


\section{Inflation}

In this section, we will briefly discuss whether cosmic strings studied in this paper can be formed after inflation. For concreteness, we will consider the inflationary model of [24]. One should also be able to extend this discussion to another heterotic M-theory inflation studied in 25$]$.

To the system of moduli in eq. (3.1), we add another five-brane multiplet corresponding to a five-brane wrapped on a non-isolated genus zero or higher genus holomorphic curve. Moduli of such a five-brane will not have any non-perturbative superpotential. Instead, the translational modulus has a potential with inflationary properties ${ }^{4}$. The process of inflation is represented by such a five-brane approaching the visible brane. It was argued in 24] that when the five-brane comes close to the visible sector, the transition vector bundle moduli [29, 30] become tachyonic and terminate inflation. Eventually, the five-brane disappears through the small instanton transition which modifies the vector bundle on the visible brane, in particular, new vector bundle moduli are created which can be stabilized by non-perturbative effects. Thus, formation of macroscopic open membranes with a small length should be related to this change of the vector bundle.

Since after the small instanton transition, the vector bundle, the number of the vector bundle moduli and the Pfaffian change, the position of the five-brane discussed in section 3 will also change. This could explain why it is stabilized close to the visible sector only after inflation. For example, let the bundle before inflation not have any moduli. Then from discussion in section 3 it follows that no minimum for the five-brane close to the visible sector will exist. Therefore, in this case, the five-brane will be stabilized at a generic point along the interval. After inflation, vector bundle moduli will be created and, according to our discussion in section 3, they might force the five-brane to move closer to the visible sector so that eq. (2.2) is satisfied. The presence of vector bundle moduli might also stabilize a five-brane close to the visible brane after inflation in the model proposed in [25]. Thus, it might be possible to create cosmic strings in the inflationary scenario in [25]. It would be interesting to understand it in detail.

\footnotetext{
${ }^{4}$ In [24], the extra five-brane multiplet had a superpotential due to a gaugino condensate in the hidden sector. In this paper, we are assuming that this superpotential is zero because it would also be a potential for the axion. However, performing the same analysis as in 24], it is not hard to show that in the absence of the gaugino condensation superpotential, the potential for the inflaton will be exactly of the same form as in [24].
} 


\section{Acknowledgments}

The author is very grateful to Juan Maldacena for lots of helpful discussions, explanations and comments on the preliminary version of the paper. The author would also like to thank Willy Fischler for discussions. The work is supported by NSF grant PHY-0070928.

\section{References}

[1] E. Witten, "Cosmic Superstrings," Phys. Lett. B 153, (1985) 243.

[2] A. Vilenkin and A. E. Everett, "Cosmic Strings and Domain Walls in Models with Goldstone and Pseudogoldstone Bosons," Phys. Rev. Lett. 48 (1982) 1867.

[3] S. Sarangi and S.-H. H. Tye, "Cosmic String Production Towards the End of Brane Inflation," Phys.Lett. B536 (2002) 185-192 hep-th/0204074.

[4] N. T. Jones, H. Stoica and S.-H. H. Tye, "The Production, Spectrum and Evolution of Cosmic Strings in Brane Inflation," Phys.Lett. B563 (2003) 6-14 hep-th/0303269.

[5] G. Dvali and A. Vilenkin, "Formation and evolution of cosmic D-strings," JCAP 0403 (2004) 010 hep-th/0312007.

[6] E. J. Copeland, R. C. Myers and J. Polchinski, "Cosmic F- and D-strings," JHEP 0406 (2004) 013 hep-th/0312067.

[7] I. R. Klebanov and M. J. Strassler, "Supergravity and a Confining Gauge Theory: Duality Cascades and $\chi$ SB-Resolution of Naked Singularities," JHEP 0008 (2000) 052 hep-th/0007191.

[8] S. Kachru, R. Kallosh, A. Linde, J. Maldacena, L. McAllister and S. P. Trivedi, "Towards Inflation in String Theory," JCAP 0310 (2003) 013 hep-th/0308055.

[9] S. Kachru, R. Kallosh, A. Linde and S. P. Trivedi, "de Sitter Vacua in String Theory," Phys.Rev. D68 (2003) 046005 hep-th/0301240.

[10] G. Dvali, R. Kallosh and A. Van Proeyen, "D-term strings," JHEP 0401 (2004) 035 hep-th/0312005.

[11] P. Binetruy, G. Dvali, R. Kallosh and A. Van Proeyen, "Fayet-Iliopoulos Terms in Supergravity and Cosmology," Class.Quant.Grav. 21 (2004) 3137-3170 hep-th/0402046. 
[12] J. J. Blanco-Pillado, G. Dvali and M. Redi, "Cosmic D-strings as Axionic D-term Strings," hep-th/0505172.

[13] S. S. Gubser, C. P. Herzog and I. R. Klebanov, "Symmetry Breaking and Axionic Strings in the Warped Deformed Conifold," JHEP 0409 (2004) 036 hep-th/0405282.

[14] P. Horava and E. Witten, "Heterotic and Type I String Dynamics from Eleven Dimensions," Nucl.Phys. B460 (1996) 506-524 hep-th/9510209.

[15] P. Horava and E. Witten, "Eleven-Dimensional Supergravity on a Manifold with Boundary," Nucl.Phys. B475 (1996) 94-114 hep-th/9603142.

[16] E. Witten, "Strong Coupling Expansion Of Calabi-Yau Compactification," Nucl.Phys. B471 (1996) 135-158 hep-th/9602070.

[17] V. Braun, Y.-H. He, B. A. Ovrut and T. Pantev, "A Heterotic Standard Model," hep-th/0501070.

[18] V. Braun, Y.-H. He, B. A. Ovrut and T. Pantev, "A Standard Model from the E8 x E8 Heterotic Superstring," hep-th/0502155.

[19] G. Curio and A. Krause, "G-Fluxes and Non-Perturbative Stabilisation of Heterotic M-Theory," Nucl.Phys. B643 (2002) 131-156 hep-th/0108220.

[20] E. I. Buchbinder and B. A. Ovrut, "Vacuum Stability in Heterotic M-Theory," Phys.Rev. D69 (2004) 086010 hep-th/0310112.

[21] S. Gukov, S. Kachru, X. Liu and L. McAllister, "Heterotic Moduli Stabilization with Fractional Chern-Simons Invariants," Phys.Rev. D69 (2004) 086008 hep-th/0310159.

[22] M. Becker, G. Curio and A. Krause, "De Sitter Vacua from Heterotic M-Theory," Nucl.Phys. B693 (2004) 223-260 hep-th/0403027.

[23] E. I. Buchbinder, "Raising Anti de Sitter Vacua to de Sitter Vacua in Heterotic MTheory," Phys.Rev. D70 (2004) 066008 hep-th/0406101.

[24] E. I. Buchbinder, "Five-Brane Dynamics and Inflation in Heterotic M-Theory," Nucl.Phys. B711 (2005) 314-344 hep-th/0411062.

[25] K. Becker, M. Becker and A. Krause, "M-Theory Inflation from Multi M5-Brane Dynamics," Nucl.Phys. B715 (2005) 349-371 hep-th/0501130. 
[26] M. Dine, N. Seiberg and E. Witten, Fayet-Illiopulos Terms in String Theory, Nucl. Phys. B 289 (1987) 589.

[27] A. Lukas, B. A. Ovrut and D. Waldram, "On the Four-Dimensional Effective Action of Strongly Coupled Heterotic String Theory," Nucl.Phys. B532 (1998) 43-82 hep-th/9710208.

[28] A. Lukas, B. A. Ovrut, K. S. Stelle and D. Waldram, "Heterotic M-theory in Five Dimensions," Nucl.Phys. B552 (1999) 246-290. hep-th/9806051.

[29] E. Buchbinder, R. Donagi and B. A. Ovrut, "Vector Bundle Moduli and Small Instanton Transitions," JHEP 0206 (2002) 054 hep-th/0202084.

[30] E. I. Buchbinder, B. A. Ovrut and R. Reinbacher, "Instanton Moduli in String Theory," JHEP 0504 (2005) 008 hep-th/0410200.

[31] E. Witten, "Small Instantons in String Theory," Nucl.Phys. B460 (1996) 541-559 hep-th/9511030.

[32] N. Seiberg and E. Witten, "Comments on String Dynamics in Six Dimensions," Nucl.Phys. B471 (1996) 121-134 hep-th/9603003.

[33] B. A. Ovrut, T. Pantev and J. Park, "Small Instanton Transitions in Heterotic MTheory," JHEP 0005 (2000) 045 hep-th/0001133.

[34] A. Lukas, B. A. Ovrut and D. Waldram, "Non-standard embedding and five-branes in heterotic M-Theory," Phys.Rev. D59 (1999) 106005 hep-th/9808101.

[35] T. W. B. Kibble, "Topology of Cosmic Domains and Strings," J. Phys. A 9 (1976) 1387.

[36] O. J. Ganor and A. Hanany, "Small $E_{8}$ Instantons and Tensionless Non-critical Strings," Nucl.Phys. B474 (1996) 122-140 hep-th/9602120.

[37] P. Yi, "Membranes from Five-Branes and Fundamental Strings from Dp Branes," Nucl.Phys. B550 (1999) 214-224 hep-th/9901159.

[38] E. Lima, B. A. Ovrut, J. Park and R. Reinbacher, "Non-Perturbative Superpotentials from Membrane Instantons in Heterotic M-Theory," Nucl.Phys. B614 (2001) 117-170 hep-th/0101049. 
[39] E. Lima, B. A. Ovrut and J. Park, "Five-Brane Superpotentials in Heterotic M-Theory," Nucl.Phys. B626 (2002) 113-164 hep-th/0102046.

[40] J. Polchinski, "Introduction to Cosmic F- and D-Strings," Lectures presented at the 2004 Cargese Summer School, hep-th/0412244.

[41] J.-P. Derendinger and R. Sauser, "A Five-brane Modulus in the Effective N=1 Supergravity of M-Theory," Nucl.Phys. B598 (2001) 87-114 hep-th/0009054.

[42] S. Gukov, C. Vafa and E. Witten, "CFT's From Calabi-Yau Four-folds," Nucl.Phys. B584 (2000) 69-108; Erratum-ibid. B608 (2001) 477-478 hep-th/9906070.

[43] M. Becker and D. Constantin, "A Note on Flux Induced Superpotentials in String Theory," JHEP 0308 (2003) 015 hep-th/0210131.

[44] E. Witten, "Non-Perturbative Superpotentials In String Theory," Nucl.Phys. B474 (1996) 343-360 hep-th/9604030.

[45] K. Becker, M. Becker and A. Strominger, "Fivebranes, Membranes and NonPerturbative String Theory," Nucl.Phys. B456 (1995) 130-152 hep-th/9507158.

[46] G. Moore, G. Peradze and N. Saulina, "Instabilities in heterotic M-theory induced by open membrane instantons," Nucl.Phys. B607 (2001) 117-154 hep-th/0012104.

[47] E. I. Buchbinder, R. Donagi and B. A. Ovrut, "Superpotentials for Vector Bundle Moduli," Nucl.Phys. B653 (2003) 400-420 hep-th/0205190.

[48] E. I. Buchbinder, R. Donagi and B. A. Ovrut, "Vector Bundle Moduli Superpotentials in Heterotic Superstrings and M-Theory," JHEP 0207 (2002) 066 hep-th/0206203.

[49] See, N. Dorey, T. J. Hollowood, V. V. Khoze and M. P. Mattis, "The Calculus of Many Instantons," Phys.Rept. 371 (2002) 231-459 hep-th/0206063, and refernces therein.

[50] J. Gray and A. Lukas, "Gauge Five Brane Moduli In Four-Dimensional Heterotic Models," Phys.Rev. D70 (2004) 086003 hep-th/0309096.

[51] J. Gray, "An explicit example of a moduli driven phase transition in heterotic models," hep-th/0406241.

[52] C. P. Burgess, R. Kallosh and F. Quevedo, "de Sitter String Vacua from Supersymmetric D-terms," JHEP 0310 (2003) 056 hep-th/0309187. 
[53] V. Balasubramanian and P. Berglund, "Stringy corrections to Kahler potentials, SUSY breaking, and the cosmological constant problem," JHEP 0411 (2004) 085 hep-th/0408054.

[54] K. Bobkov, "Volume Stabilization via $\alpha$ Corrections in Type IIB Theory with Fluxes," hep-th/0412239.

[55] V. Balasubramanian, P. Berglund, J. P. Conlon and F. Quevedo, "Systematics of Moduli Stabilisation in Calabi-Yau Flux Compactifications," JHEP 0503 (2005) 007 hep-th/0502058].

[56] A. Saltman and E. Silverstein, "A New Handle on de Sitter Compactifications", hep-th/0411271. 